\title{
ANÁLISE DOS RESULTADOS INICIAIS DO PROGRAMA DE TRANSPLANTE RENAL INTERVIVOS NO ESTADO DO PARÁ
}

\author{
Analysis of the initial results on the live donor kidney transplantation program in the State of Para
}

\begin{abstract}
Aluízio Gonçalves da Fonseca, Silvia Cruz Mignone, José Ricardo Tuma da Ponte, Sidney Antonio Cruz, Fernando Jordão de Souza, Paulo Martins Toscano, Mauro Ferreira de Almeida, Simone Martins Lima
\end{abstract}

\begin{abstract}
RESUMO
Objetivo: Avaliar os resultados iniciais deste programa, assim como identificar os principais fatores de risco relacionados com a perda do enxerto. Métodos: Foram avaliados retrospectivamente 65 transplantes renais intervivos, quanto à sobrevida do enxerto e sobrevida dos pacientes de um, três e cinco anos. Estas serviram como ponto de corte para a análise da importância de vários fatores de risco do doador e receptor para perda do enxerto. Para análise das sobrevidas foi utilizado o método de Kaplan-Meier. Os modelos de regressão univariado e multivariado de Cox foram utilizados para os fatores de risco. Resultados: As sobrevidas do enxerto e dos pacientes de um, três e cinco anos foram respectivamente $98 \%, 91 \%$ e $83 \%$; $98 \%, 95 \%$ e $87 \%$. O óbito com enxerto funcional ocorreu em $60 \%$ dos pacientes. A análise univariada dos fatores demonstrou em ordem decrescente de importância que o número de transfusões, sorologia positiva para hepatite e citomegalovírus, doença de base, tipo de imunossupressão e tempo de diálise foram significativos para a perda do enxerto. A análise multivariada não mostrou qualquer significância estatística. Conclusões: Os resultados iniciais deste programa mostraram-se satisfatórios. Os óbitos por causas não relacionadas ao transplante representaram causa importante de perda do enxerto. Medidas no sentido de melhorar a avaliação clínica pré-operatória e atuar nos fatores de risco devem ser enfatizadas, visando melhorar os resultados do transplante renal intervivos em longo prazo.
\end{abstract}

Descritores: Transplante renal, mortalidade, Sobrevivência, Fatores de Risco, Sobrevivência de Enxerto.

\section{Instituição:}

Departamento de Uronefrologia do Hospital Ofir Loiola

Av. Magalhães Barata, 992

CEP 66063-240 - Belém - Pará

Tel./Fax: 5591 3289-1000

Correspondência:

Aluízio Gonçalves da Fonseca

Tv. Dom Romualdo de Seixas, 1529 - Umarizal

CEP 66055-200 - Belém - Pará

Tel./Fax: 5591 3223-3878

E-mail: agf@supridados.com.br

Recebido em: 29.06.2005
Aceito em: 30.03 .2007

\section{INTRODUÇÃO}

O transplante renal é o tratamento de escolha para pacientes com insuficiência renal crônica, apresentando vantagens significativas em relação aos pacientes em diálise, principalmente na esfera psicológica e emocional, oferecendo inclusive a possibilidade de retorno ao trabalho. ${ }^{1-4}$

$\mathrm{Na}$ atualidade, o número ainda pequeno de órgãos provenientes de doadores falecidos tem tornado o transplante renal intervivos a forma mais viável de suprir as necessidades dos pacientes em fila de espera. ${ }^{4,5}$

Os resultados em curto prazo sofreram grande melhora com o advento de novos esquemas de imunossupressão. Todavia os resultados tardios ainda apresentam índices elevados de perda do enxerto. ${ }^{6}$ A literatura tem reportado inúmeros fatores prognósticos que podem afetar a sobrevida do enxerto e dos pacientes submetidos a transplante renal de doadores vivos. ${ }^{7-16}$

O primeiro transplante renal intervivos realizado no Estado do Pará ocorreu em Agosto de 1999. A implantação deste programa foi motivada pela necessidade de oferecer tratamento adequado à enorme demanda de pacientes com doença renal crônica no estado.

A proposta do presente estudo foi avaliar os resultados iniciais daquele programa, assim como os fatores de risco, associados com a perda do enxerto. 


\section{MÉTODO}

De agosto de 1999 a Julho de 2004 foram realizados 65 transplantes renais intervivos no Departamento de Uronefrologia do Hospital Ophir Loyola, Belém, Pará, todos analisados retrospectivamente. O seguimento variou de 6 a 60 meses (média de $33 \pm 38$ meses). Entre os doadores, 26 eram do sexo masculino e 39 do feminino, com média de idade de $36 \pm 10$ anos. Entre os receptores havia 41 homens e 24 mulheres, com média de idade de 36,1 \pm 14 anos. Estes receberam enxertos de 55 doadores relacionados e de 10 não relacionados. Apenas um paciente foi submetido a segundo transplante. Imunossupressão tríplice (ciclosporina, azatioprina e prednisona) foi utilizada em 49 pacientes, enquanto a utilização de micofenolato mofetil foi adicionada em 16 pacientes, em substituição à azatioprina.

Além dos dados demográficos descritos acima, outras variáveis e co-variáveis clínicas pré, trans e pós-operatórias, relacionadas aos doadores e receptores foram analisadas (tabelas 1, 2 e 3).

Doadores: As variáveis clínicas relacionadas aos doadores incluíram incompatibilidade HLA (0,1 a 2, 3 a 4 e 5 a 6), Rim doado (esquerdo/direito), cirurgia de banco (reconstrução ex vivo dos vasos renais, sim/não), 1o tempo de isquemia quente (tempo entre o clampeamento da artéria renal até o início da perfusão em banco), $2^{2}$ tempo de isquemia quente (tempo entre o início da anastomose vascular até a reperfusão do órgão) e tempo de isquemia

Tabela 1: Variáveis do doador e análise estatística univariada e multivariada pelo modelo de risco proporcional de Cox.

\begin{tabular}{cccc}
\hline & & \multicolumn{2}{c}{ Análise estatística } \\
\hline & & Univariada & Multivariada \\
\hline Variáveis do doador & $n(65)$ & $\boldsymbol{p}$ & $\boldsymbol{p}$ \\
\hline Sexo M/F & $26 / 39$ & 0,37 & 0,17 \\
Idade & $36 \pm 10,4(20-58)$ & 0,66 & 0,12 \\
DVR./DVNR. & $55 / 10$ & 0,20 & 0,25 \\
Incompatibilidade HLA & & & \\
0 & 6 & 0,57 & 0,39 \\
1 a 2 & 18 & 0,25 & 0,83 \\
3 a 4 & 32 & 0,79 & 0,13 \\
5 a 6 & 9 & 0,30 & 0,11 \\
Rim doado E/D & $60 / 5$ & 0,76 & 0,68 \\
Cirurgia de banco S/N & $4 / 61$ & 0,49 & 0,43 \\
10 TIQ(min) & $2,6 \pm 1,4(4-6)$ & $\mathbf{0 , 0 3}$ & 0,12 \\
20 TIQ(min) & $47,7 \pm 12,3(30-108)$ & 0,90 & 0,56 \\
TIT(min) & $48,9 \pm 12,4(30,2-108,4)$ & 0,79 & 0,61 \\
\hline
\end{tabular}

Abreviaturas: M/F: masculino/feminino; DVR/DVNR: doador vivo relacionado/doador vivo não relacionado; HLA: antígeno leucocitário humano; E/D: esquerdo/direito; S/N: sim/não; TIQ: tempo de isquemia quente; $\boldsymbol{p}$ : valor $\boldsymbol{p}(<0,05)$. total (soma de ambos os anteriores) (tabela 1).

Receptores: As variáveis clínicas relacionadas aos receptores incluíram doença de base (GNC, urológicas, doença policística, nefropatia diabética, doenças sistêmicas e indeterminadas), tempo de diálise (meses), sorologia (negativa, CMV, hepatite $\mathrm{B}$ ou $\mathrm{C}$, $\mathrm{CMV}+$ hepatite $\mathrm{B}$ ou $\mathrm{C}, \mathrm{CMV}+$ toxoplasmose), creatinina prétransplante, transfusões pré-transplante $(0,1$ a 5,6 a 10,11 a $15,>$ 15), complicações cirúrgicas, imunossupressão, rejeição aguda e crônica (tabelas 2 e 3 ).

Análise estatística: A sobrevida do enxerto foi definida como receptores vivos com o enxerto funcional e, como desfecho,

Tabela 2: Variáveis do receptor e análise estatística pelo modelo de risco proporcional de Cox.

Análise estatística

\begin{tabular}{|c|c|c|c|}
\hline & & \multicolumn{2}{|c|}{ Análise estatística } \\
\hline & & Univariada & Multivariada \\
\hline Variáveis do receptor & $n(65)$ & $P$ & $P$ \\
\hline \multicolumn{4}{|l|}{ Doença de base } \\
\hline GNC & 36 & 0,04 & 0,10 \\
\hline Urológicas & 2 & 0,12 & 0,29 \\
\hline Dç. Policística & 2 & 0,85 & 0,70 \\
\hline Nefropatia diabética & 7 & 0,57 & 0,99 \\
\hline Dçs. Sistêmicas & 7 & 0,11 & 0,49 \\
\hline Indeterminada & 11 & 0,22 & 0,55 \\
\hline $\begin{array}{l}\text { Tempo de diálise } \\
\text { (meses) }\end{array}$ & $20,8 \pm 15,9(3-98)$ & 0,002 & 0,08 \\
\hline \multicolumn{4}{|l|}{ Sorologia } \\
\hline Negativa & 15 & 0,77 & 0,69 \\
\hline CMV & 40 & 0,22 & 0,29 \\
\hline Hept. B ou C & 3 & 0,75 & 0,41 \\
\hline CMV+Hept. B ou C & 5 & 0,05 & 0,10 \\
\hline CMV+Toxopl. & 2 & 0,24 & 0,20 \\
\hline \multicolumn{4}{|l|}{ Transfusões pré Tx } \\
\hline 0 & 10 & 0,37 & 0,52 \\
\hline 1 a 5 & 42 & 0,36 & 0,30 \\
\hline 6 a 10 & 9 & 0,98 & 0,94 \\
\hline 11 a 15 & 2 & 0,07 & 0,53 \\
\hline$>15$ & 2 & 0,04 & 0,42 \\
\hline Imunossupressão C/0 & $49 / 16$ & 0,03 & 0,26 \\
\hline
\end{tabular}

Abreviaturas: GNC: glomerulonefrite crônica; CMV: citomegalovírus; TX: transplante; Hepat.: hepatite; Toxopl.: toxoplasmose; C/O: clássical outras; $\boldsymbol{p}$ : valor $\boldsymbol{p}(<0,05)$. 
Tabela 3: Variáveis do receptor e análise estatística pelo modelo de risco proporcional de Cox.

\begin{tabular}{cccc}
\hline & & \multicolumn{2}{c}{ Análise estatística } \\
\hline Variáveis do receptor & $n(65)$ & Univariada & Multivariada \\
\hline Sexo M/F & $41 / 24$ & $\boldsymbol{p}$ & 0,19 \\
Idade & $36,1 \pm 14(11-70)$ & 0,60 & 0,50 \\
$\begin{array}{c}\text { Creatinina pré Tx } \\
\text { (ng/dl) }\end{array}$ & $9,1 \pm 2,4(3-17,7)$ & 0,28 & 0,59 \\
$\begin{array}{c}\text { Complicações } \\
\text { cirúrgicas S/N }\end{array}$ & $21 / 44$ & & 0,65 \\
$\begin{array}{c}\text { Rejeição aguda S/N } \\
\text { Rejeição crônica S/N }\end{array}$ & $23 / 42$ & 0,82 & 0,16 \\
\hline
\end{tabular}

Abreviaturas: M/F: masculino/feminino; Tx: transplante;

ng/dl: nanograma por decilitro; $S / N$ : sim/não.

foi considerado nefropatia crônica do enxerto comprovada histologicamente, com necessidade de retorno à diálise ou óbito com o enxerto funcional. A sobrevida dos pacientes foi definida como óbito por qualquer causa. A análise da influência dos fatores de risco foi realizada utilizando-se os dois pontos de corte. Na análise de sobrevida de um, três e cinco anos foi utilizado o método de Kaplan-Meier (Figura 1). A análise univariada e multivariada dos fatores de risco dos doadores e receptores foi realizada pelo modelo de regressão do risco proporcional de Cox, aceitando-se como significância estatística o valor de $p<0,05$ (tabelas 1,2 e 3). Foi utilizado o software NCSS e PASS, versão 2005, Kaysville, Utha.

Figura 1: Sobrevidas do enxerto dos pacientes de 1, 3 e 5 anos em 65 transplantes renais intervivos.

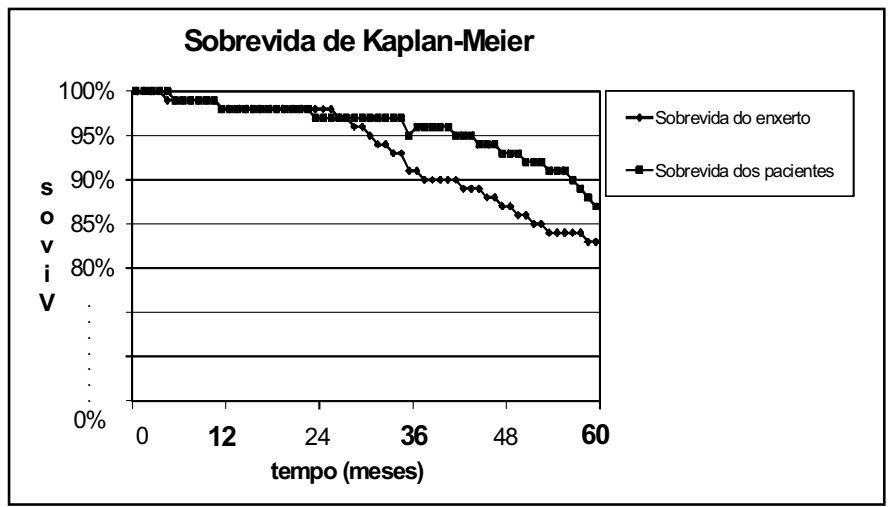

\section{RESULTADOS}

Houve dez ocorrências no grupo estudado, sendo que seis pacientes foram a óbito: dois devido a choque cardiogênico, dois por infecção, um por fístula urinária e um devido à ruptura do enxerto. Quatro pacientes perderam o enxerto devido à rejeição crônica. A sobrevida de um, três e cinco anos do enxerto foi de $98 \%$, 91\% e $83 \%$ respectivamente, e a sobrevida dos pacientes de um, três e cinco anos foi de $98 \%, 95 \%$ e $87 \%$.

Entre os fatores de risco do doador, a análise univariada demonstrou que o $1^{\mathrm{o}}$ TIQ $(p: 0,03)$ foi significativo para a perda do enxerto, enquanto na análise multivariada nenhum fator demonstrou significância estatística independente.

Entre os fatores de risco do receptor, a análise univariada demonstrou que o número de transfusões $(p: 0,04)$, sorologia positiva para $\mathrm{CMV}+$ hepatite $\mathrm{B}$ ou $\mathrm{C}(p: 0,04)$, glomerulonefrite crônica $(p: 0,04)$, esquema de imunossupressão utilizado $(p: 0,03)$ e tempo de diálise $(p: 0,002)$ mostraram-se significativos para perda do enxerto, enquanto na análise multivariada nenhum dos fatores de risco demonstrou significância. Não houve diferenças entre análises, utilizando-se a sobrevida do enxerto e sobrevida dos pacientes separadamente como ponto de corte para análise da influência dos fatores de risco.

\section{DISCUSSÃO}

A maioria dos autores considera os óbitos como perda do enxerto; entretanto, este modelo de análise pode superestimar os efeitos dos fatores de risco avaliados e alterar os resultados obtidos. ${ }^{17-20}$

Os óbitos foram responsáveis por $60 \%$ dos pacientes censurados no presente estudo; por este motivo, procuramos analisar nossos dados utilizando os dois índices de sobrevida. Todavia, não houve diferenças nos resultados, fato que parece estar relacionado ao pequeno número de pacientes e ocorrências avaliados.

As sobrevidas do enxerto e dos pacientes em um, três e cinco anos comparam-se favoravelmente com as sobrevidas de um e cinco anos de $92 \%, 76 \%$ e $97 \%, 91 \%$ respectivamente, descritas por outros autores. ${ }^{16}$

Óbitos por causas diversas, não relacionadas ao transplante, são as principais causas não imunológicas de perda do enxerto, segundo registros da UNOS (United Network for Organ Sharing). Esta causa contribuiu com $23 \%$ dos casos no primeiro ano, e 35\% após esse período. ${ }^{16} \mathrm{Na}$ Europa e nos Estados Unidos, a doença coronariana é a principal causa dessa ocorrência, seguida de sepse, câncer e acidente vascular cerebral. Além disso, houve aumento de $14 \%$ nos anos 70 para 31\% na década de 90 dos óbitos devido àquela causa. ${ }^{19,20}$ Quanto aos óbitos por sepse, observa-se redução de $43 \%$ para $27 \%$ na última década. ${ }^{20}$ Segundo a UNOS, essa foi a causa de falha do transplante em $3 \%$ no primeiro ano e $1 \%$ após. ${ }^{16}$

$\mathrm{Na}$ análise estatística, procurou-se avaliar a importância de inúmeras variáveis independentes dos doadores e receptores na falha do transplante, adotando como ponto de corte para a sobrevida do enxerto e dos pacientes.

Entre as variáveis dos doadores, apenas o primeiro tempo de isquemia quente (1ำ TIQ) mostrou-se significativo na análise univariada. Essa variável não tem apresentado importância na literatura, e, apesar de não ter apresentado importância independente em um trabalho no qual esse fator foi importante, os valores registrados em minutos do 1으, TIQ, mostraram-se inferiores aos desta amostra. Este fato pode estar relacionado com a curva de aprendizado no início do programa. ${ }^{21}$

$\mathrm{Na}$ análise das variáveis dos receptores, a análise univariada mostrou em ordem decrescente em importância que o número de transfusões, sorologia positiva para hepatite e citomegalovírus, 
doença de base, tipo de imunossupressão e tempo de diálise influenciaram negativamente nas sobrevidas avaliadas, apesar de nenhum deles ter mostrado força independente, o que sugere que a perda do enxerto seja influenciada por múltiplos fatores de risco.

A utilização sistemática de transfusões sanguíneas como parte dos protocolos de imunossupressão foi utilizada até 1985. Após esse período, os poucos benefícios observados, além dos riscos potenciais de doenças infecto-contagiosas e melhora dos protocolos de imunossupressão desestimularam essa prática. ${ }^{22-25}$ A resposta imunológica às transfusões sanguíneas prévias ao transplante pode ser sensibilização ou tolerância. Em pacientes expostos previamente a aloantígenos em decorrência de transplantes anteriores, gestações múltiplas e transfusões sanguíneas maciças, podem desenvolver anticorpos anti-HLA. Por outro lado, nos pacientes não submetidos a estas condições, as transfusões têm sido associadas à redução da resposta imune e melhor sobrevida do enxerto. ${ }^{24}$ Portanto, tal efeito paradoxal, faz com que essa questão esteja longe de ser resolvida. Os consensos atuais procuram evitar a sensibilização por transfusões múltiplas. ${ }^{26}$ Em nossa amostra, todos os pacientes que sofreram rejeição crônica, receberam transfusões prévias, em média seis unidades de concentrado de hemáceas, fato que pode ser explicado pelo grande tempo de espera antes que o programa regional de transplantes fosse implantado. O risco relativo para perda do enxerto mostrou-se progressivamente maior com o aumento do número de transfusões, alcançando pico na faixa de 11 a 15 unidades de hemoderivados. Portanto, parece aconselhável evitar transfusões, principalmente em pacientes com sensibilização maciça prévia.

A sorologia positiva para Hepatite $\mathrm{B}$ ou $\mathrm{C}$ e Citomegalovírus representou fator de risco significativo para perda do enxerto. Alguns autores encontraram a mesma tendência em pacientes com sorologia para Citomegalovírus positiva; no entanto, com a exclusão dos pacientes que foram a óbito com o enxerto funcional a mesma perdeu significado, indicando que o resultado pode ter sofrido influência. ${ }^{27}$ Por outro lado, outros autores não encontraram relação positiva nessa associação após seguimento de cinco anos. ${ }^{28}$ A sorologia positiva para vírus da hepatite $\mathrm{B}$ e $\mathrm{C}$ pode influenciar negativamente a sobrevida de dez anos dos pacientes transplantados; todavia, essa tendência não ocorre nos casos de infecção isolada por um deles. ${ }^{29}$ Estudos com um número maior de pacientes avaliando a associação de agentes infecciosos como fator de risco para perda do enxerto devem ser realizados.

Em nossa casuística, o diagnóstico de glomerulonefrite como doença de base no receptor apresentou significância estatística para perda do enxerto na análise univariada. Esse risco parece estar relacionado com a recidiva da doença no rim transplantado. ${ }^{30} \mathrm{Os}$ mecanismos fisiopatológicos responsáveis pela recidiva da doença primária não são bem conhecidos, assim como poucos trabalhos têm abordado esse assunto. ${ }^{30,31}$ A prevalência de recorrência da doença primária varia entre 1,8 e $6,5 \% .{ }^{30}$ Parece haver uma variação na prevalência de perda do enxerto entre as várias glomerulopatias, sendo mais prevalente na glomeruloesclerose focal segmentar, seguida de nefropatia por $\operatorname{IgA}$, glomerulonefrite membranoproliferativa e nefropatia diabética. ${ }^{16,30,31}$ Essa recorrência raramente ocorre antes de dois anos pós-transplante. ${ }^{30}$

Em relação à imunossupressão, a grande maioria dos pacientes foi tratada com o esquema clássico, incluindo ciclosporina, azatioprina e prednisona, que é utilizado em inúmeros centros de transplante; por isso, não foi possível identificar o motivo de tal significância estatística. ${ }^{1-15}$ Todavia, existe a possibilidade de que os baixos níveis séricos de ciclosporina associados à baixa aderência dos pacientes possam estar envolvidos. ${ }^{30}$ Outra possibilidade é que possa ter havido interação com outras variáveis como a incompatibilidade do HLA, que estava na faixa de três a quatro em $70 \%$ dos pacientes que sofreram falência do enxerto. Apesar da falta de significância isolada neste estudo, este fator pode interferir negativamente na sobrevida do enxerto, segundo vários autores. ${ }^{16,29-31}$

O tempo de diálise pré-transplante associou-se negativamente à sobrevida o enxerto. Segundo alguns autores, quanto maior o tempo de diálise, pior a sobrevida do enxerto. ${ }^{16,29-32}$ Períodos maiores que seis meses têm sido associados com significativo e progressivo risco de perda do enxerto. ${ }^{16,30}$ Pacientes submetidos a transplante sem diálise prévia apresentaram melhor sobrevida do enxerto, com redução dos índices de falência de $86 \%$ no terceiro ano de transplante. ${ }^{32}$ Por ser o único centro de transplante da região e encontrar-se em período recente de implantação, é compreensível que a maioria dos pacientes estejam em espera por longo tempo.

\section{CONCLUSÃO}

A avaliação dos resultados iniciais deste programa de transplante renal demonstra sobrevidas do enxerto e dos pacientes bastante satisfatórias. O óbito por causas não relacionadas ao transplante ainda representa importante causa de perda do enxerto. Entre os fatores de risco, transfusões sanguíneas, sorologia positiva para hepatite e citomegalovírus, glomerulonefrite, esquema de imunossupressão e tempo de diálise mostraram-se significativos para perda do enxerto. Por isso, medidas no sentido de melhorar a avaliação clínica pré-operatória e atuar nos fatores de risco devem ser enfatizadas, visando melhorar os resultados de longo prazo do transplante renal intervivos.

\section{ABSTRACT}

Purpose: To assess the initial results of this program, as well as identifying the main risk factors related to the graft loss. Methods: 65 living donor kidney transplantations have been retrospectively assessed as to the graft, patients, and total survival in 1,3 , and 5 years. These served as cut-point for the analysis on the importance of many donor and receptor risk factors for the graft loss. To assess the survival, the Kaplan-Meier method was used. The univariate and multivariate Cox regression models were used for the risk factors. Results: The graft survival and patients in 1, 3, and 5 years have been respectively $98 \%, 91 \%$, and $83 \%$; $98 \%$, 95\%, and $87 \%$. The death with good functional graft occurred in $60 \%$ patients. The univariate analysis of the risk factors showed in decreasing importance that the amount of transfusions, positive serology for hepatitis and cytomegalovirus as base disease, kind of immunosuppressant, and extent of the dialysis were significant for the graft loss. The multivariate analysis showed no statistic significance. Conclusions: The initial results of this program revealed to be satisfactory. Death due to reasons that were not transplant-related represented an important cause 
to the graft loss. Measures aimed to improve the clinic preoperative assessment and acting on the risk factors must be emphasized, seeking to improve the late results of the live donor kidney transplantations.

Keywords: Kidney Transplantation, mortality, Survival, Risk Factors, Graft Survival.

\section{REFERÊNCIAS}

1. Haberal M, Emiroglu R, Moray G, Karakali H, Arslan G. Living-Donor kidney transplantation: Single center experience. Transplant Proc 2002; 34:2056-59.

2. Thulo TP, Mobida MCM, Kobrin A, Ndlovu S, Becker P. Five-Year results of living related renal donation are similar to cadaveric transplantation in black south africans. Transplant Proc. 2002;34:2565-6.

3. Vareesangthip K, Jitpraphai P, Vongwiwatana A, Permpikul P, Pornpong C, Larpkitkachorm R. Results of renal transplantation of the Siriraj Hospital-Thailand. Transplant Proc. 2003;35:159-62.

4. Michelon T, Piovesan F, Santos P, Keitel E, Bittar A, Neumann J et al. Impact of using marginal donors and long cold ischemia time in renal transplant survival. Transplant Proc. 2000;32:2586-8.

5. He X, Johnston A. Early acute rejection does not affect chronic allograft nephropathy and death censored graft failure. Transplant Proc 2004; 36:2993-96.

6. Kwon OJ, Kwak JY. The Impact of sex and age matching for long-term graft survival in living donor renal transplantation. Transplant Proc. 2004;36:2040-2.

7. OhCK, Kim SJ, Kim JH, Shin GT, Kim HS. Influence of donor and recipient gender on early graft function after living donor kidney transplantation. Transplant Proc. 2004;36:2015-7.

8. Haberal M, Emiroglu R, Yagmurdur MC, Karakayali H, Moray G, Arslan G et al. Results with living-Donor kidney transplants from spouses: Fourteen years of experience at our center. Transplant Proc. 2002;34:2410-1.

9. Park YH, Lee JN, Min SK, Lee WK, Lee YD. Comparative results of kidney transplantation from living donors. Transplant Proc. 2002;35:156-7.

10. Park YH, Lee JN, Min SK, Lee WK, Joo KW, Cha MK et al. Review of 95 consecutive kidney transplantation in one center. Transplant Proc. 2003;35:145-6.

11. Haberal M, Emiroglu R, Arslan G, Karakayali H, Moray G, Bilgin N. A retrospective analysis of 1000 renal transplantation performed at one center. Transplant Proc. 2002;34:2405-7.

12. Ponticelli C, Villa M, Cesana B, Montagnino G, Tarantino A. Risk factors for late allograft failure. Kidney Int. 2002;62:1848-54.

13. Sirivongs D, Liawnoraset W, Pongskul C, Reungjui S. Graft survival analysis in kidney transplantion: A 12-year experience in a Thai Medical Center. Transplant Proc. 2004;36:2034-7.

14. Kim SJ, Lee HH, Lee DS, Lee KW, Joh JW, Woo DH et al. Prognostic factors affecting graft and patient survival in cadaveric and living kidney transplantation. Transplant Proc. 2004;36:2038-9.

15. Xiao X, Li Y, Ao J, Chen Y. Analysis of prognostic factors affecting renal allograft survival. Transplant Proc. 1992;24:1442-6.

16. Cecka M. Clinical outcome of renal transplantation: Factors influencing patient and graft survival. Surg Clin North Am. 1998 78:133-47.

17. Pessione F, Cohen S, Durand D, Hourmant M, Kessler M, Legendre C et al. Multivariate analysis of donor risk factors for graft survival in kidney transplantation. Transplantation. 2003;75:361-7.
18. Hetzel GR, Klein B, Brause M, Westhoff A, Willers R, Sandmann W. Risk factors for delayed graft function after renal transplantation and their significance for longterm clinical outcome. Transplant Int. 2002;15:10-6.

19. Yoshimura N, Oka Y, Nakane I, Aikawa M, Okamoto M, Akioka K, et al. Long-Term results and complications of living related kidney transplantation in a single center. Transplant Proc. 2002;34:1675-7.

20. Howard RJ, Reed AI, Hemming AW, Van der Werf WJ, Patton PR, Pfaff WW et al. Graft loss and death: Changing causes after kidney transplantation. Transplant Proc. 2001;33:3416.

21. Roodnat JI, Mulder PGH, Van Riemsdijk IC, Ijzermans JNM, Van Gelder T, Weimar W. Ischemia times and donor serum creatinine in relation to renal graft failure. Transplantation. 2003;75:799-804

22. Scornik JC, Ireland JE, Howard RJ, Pfaff W. Assessment of the risk for broad sensitization by blood transfusions. Transplantation. 1984;37:249-53.

23. Pfaff WW, Fennell RS, Howard RJ, Ireland JF, Scornik JC. Planned random donor blood transfusion in preparation for transplantation: Sensitization and graft survival. Transplantation. 1984;38:701-3.

24. Scornik JC, Ireland JE, Howard RJ, Fennell RS, Pfaff WW. Role of regular and leukocyte-free blood transfusions in the generation of broad sensitization. Transplantation. 1984;38:594-8.

25. Scornik JC, Pfaff WW, Howard RJ, Fennell III RS, Ramos E, Peterson JC. Increased antibody responsiveness to blood transfusions in pediatric patients. Transplantation. 1994;58:1361-5.

26. Käble T, Lucan M, Nicita G, Sells R, Burgos Revilla FJ, Wiesel M. Guidelines on Renal Transplantation. European Urologic Association 2004; Update:6-66.

27. Fitzgerald JT, Gallay B, Taranto SE, Mcvicar JP, Troppmann C, Chen X. Pretransplant recipient cytomegalovirus seropositivity and hemodialysis are associated with decreased renal allograft and patient survival. Transplantation. 2004;77:1405-11.

28. Dickenmann MJ, Cathomas G, Steiger J, Mihotsch MJ, Thiel G, Tamm M. Cytomegalovitus Infection and graft rejection in kidney transplantation. Transplantation. 2001; 71:710-11.

29. Corrêa JRM, Rocha FD, Peres AF, Gonçalves LF, Manfro RC. Efeito a longo prazo da infecção pelo vírus das hepatites B e C na sobrevida de pacientes transplantados Renais. Rev Assoc Med Bras 2003; 49:389-94.

30. Hariraran S. Long term kidney transplant survival. Am J Kid Dis. 2001; 38 Suppl 6:S44-50.

31. Briggs JD, Jones E. Recurrence of glomerulonephritis following renal transplantation. Nephrol Dial Transplant. 1999;14:564-5.

32. Mange KC, Joffe MM, Feldman HI. Effect of the use or nonuse of long-term dialysis on the subsequent survival of renal transplants from living donors. New Engl J Med. 2001;344:726-31. 